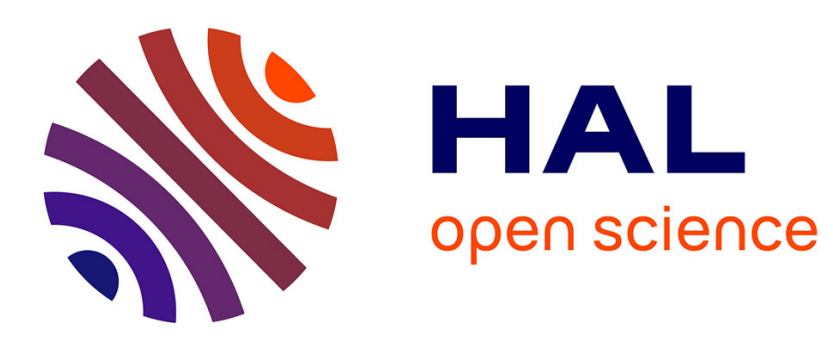

\title{
L'accès aux soins des personnes en situation de précarité
}

J. Geeraert, Elisabeth Rivollier

\section{To cite this version:}

J. Geeraert, Elisabeth Rivollier. L'accès aux soins des personnes en situation de précarité. Soins, 2014, pp.14-18. 10.1016/j.soin.2014.09.017 . halshs-01100057

\section{HAL Id: halshs-01100057 https://shs.hal.science/halshs-01100057}

Submitted on 7 Jan 2015

HAL is a multi-disciplinary open access archive for the deposit and dissemination of scientific research documents, whether they are published or not. The documents may come from teaching and research institutions in France or abroad, or from public or private research centers.
L'archive ouverte pluridisciplinaire HAL, est destinée au dépôt et à la diffusion de documents scientifiques de niveau recherche, publiés ou non, émanant des établissements d'enseignement et de recherche français ou étrangers, des laboratoires publics ou privés. 


\section{L'accès aux soins des personnes en situation de précarité}

JÉRÉMY GEERAERT ${ }^{\mathrm{a} \text {,* }}$ Sociologue, doctorant moniteur en sociologie à I'Université Paris 13, rattaché au laboratoire de l'Institut de recherche interdisciplinaire sur les enjeux sociaux (Iris)

ELISABETH RivOLLIER ${ }^{\mathrm{b}}$ Praticien hospitalier, médecin généraliste, unités de la permanence d'accès aux soins de santé et de lutte antituberculeuse, service d'urgence et de réanimation

${ }^{a} 4$, rue de Toulouse, 93600 Aulnay-sous-bois, France

${ }^{\mathrm{b}}$ Centre hospitalier universitaire, 42055 Saint-Étienne cedex 2 . France

${ }^{*}$ Auteur correspondant. Adresse e-mail : geeraert.j@gmail.com (J. Geeraert).

- L'hôpital est la porte d'entrée privilégiée d'une partie grandissante de la population en situation de précarité économique et sociale C'est pourquoi il est important que les soignants comprennent ces difficultés afin d'optimiser leurs interventions.

(C) 2014 Publié par Elsevier Masson SAS

Mots clés - accès aux soins ; accès aux droits ; assurance maladie ; coordination ; exclusion ; précarité ; travail social

\section{Summary}

(C) 2014 Published by Elsevier Masson SAS

Keywords -

\hôpital est un lieu ouvert à tous et représente, pour ceux qui sont exclus des autres structures de santé, le seul accès à des soins. Cette mission est soulignée par la loi de lutte contre les exclusions de 1998 [1] qui introduit notamment, dans les hôpitaux, des permanences d'accès aux soins de santé (Pass) [2,3] (encadré 1). Cependant, les soignants et personnels hospitaliers se retrouvent souvent dépourvus face à des demandes auxquelles ils ne savent pas répondre car jugées trop vagues ou ne relevant pas de leurs compétences.

I Le préambule de la constitution de l'organisation mondiale de la santé (OMS) définit la santé comme « un état de complet bien-être physique, mental et social [qui] ne consiste pas seulement en l'absence de maladie ou d'infirmité» [4]. Cet état intègre ainsi des déterminants sociaux, économiques, psychologiques, etc. : disposer d'un logement, pouvoir se nourrir de manière équilibrée, accéder à l'éducation.

I De nombreux travaux mettent en lumière des inégalités fortes de morbidité et de mortalité entre les classes aisées et les plus pauvres ; ces études montrent que la précarité et la pauvreté sont des facteurs

ENCADRÉ 1

Les permanences d'accès aux soins de santé

Les permanences d'accès aux soins de santé (Pass) sont issues de la loi de lutte contre les exclusions de 1998 [1] : chaque établissement public de santé a pour mission de mettre en place une cellule médico-sociale pour accueillir les personnes en situation de précarité, notamment ceux qui ne disposent pas de couverture sociale. Ce dispositif vise à faciliter leur accès au système de santé et à les accompagner dans les démarches nécessaires à la reconnaissance de leurs droits en matière de santé. II fournit soins et médicaments sans que les patients aient à avancer les frais. Les professionnels des Pass ont également une mission de sensibilisation et de formation du personnel hospitalier.

I Les Pass peuvent se présenter sous différentes formes. Elles peuvent disposer d'un lieu dédié (Pass dédiées) où assistants sociaux, médecins généralistes et infirmiers proposent un accompagnement social et médical aux patients concernés jusqu'au retour au droit commun (lorsque cela est possible). Lorsqu'elles ne disposent pas de locaux, les Pass sont dites transversales, c'est-à-dire que leurs actions s'effectuent dans tout l'hôpital en proposant un accompagnement social spécifique vers le droit commun des patients sans couverture sociale. Chaque Pass met en place des procédures pour que les patients puissent bénéficier en ambulatoire du plateau technique de l'hôpital (examens complémentaires, délivrance de médicaments, avis spécialisés).

Actuellement, plus de $\mathbf{4 0 0}$ Pass sont implantées dans les établissements de santé en France et leur développement se poursuit. 
de mauvaise santé [5]. Lutter contre ces inégalités passe donc par la réduction des écarts sociaux. Prendre en compte les spécificités en matière de santé liées à la précarité s'impose. À l'hôpital, il s'agit d'interroger l'égalité de traitement aveugle prônée par l'institution républicaine pour y introduire une prise en charge adaptée aux problématiques des populations les plus vulnérables.

\section{LA MONTÉE \\ DE LA PRÉCARITÉ \\ DANS LES SOCIÉTÉS MODERNES}

Les notions de précarité et de nouvelle pauvreté [6] apparaissent à la fin des années 1980, la crise économique se prolongeant. De nouvelles catégories sont alors touchées par la pauvreté : les chômeurs de longue durée, les jeunes peu diplômés, les mères célibataires, les personnes âgées isolées, ceux qui vivent avec des minima sociaux, les travailleurs étrangers avec ou sans papiers. S'ajoutent, dans les années 1990, les travailleurs dont les emplois se précarisent [7].

I La notion de précarité souligne le nouveau visage de la pauvreté qui dépasse la dimension strictement économique pour l'élargir à une accumulation de conditions de vie instables engendrant des difficultés diverses. Cumulées, celles-ci augmentent le risque d'une rupture progressive des liens sociaux : avec la famille, le milieu professionnel, le réseau amical, etc. La précarité est cependant considérée comme un processus transitoire et/ou réversible dans le temps [8]. Dans ses formes les plus sévères, elle se transforme en exclusion ou en grande pauvreté. Sont touchées certaines catégories de personnes très vulnérables de par leur trajectoire et/ou leur place dans la société, comme les "jeunes en galère" [9] et les "marginaux" (toxicomanes, prostituées, sortants de prison, sans-domicile-fixe, etc.). Ils doivent également faire face aux stigmates et handicaps de leur mode de vie. Il faut aussi citer les "sans-papiers", certaines communautés d'Europe, notamment les Roms, et un grand nombre d'immigrés en situation régulière qui connaissent une précarité supplémentaire de taille liée à leur statut administratif [10]. Toutes ces formes de précarité provoquent des problèmes de santé spécifiques, aggravés par des difficultés d'accès aux soins liées aux droits.

\section{Penser le corps ET LA MALADIE}

I Le rapport au corps, la manière de percevoir la maladie ou un symptôme, les représentations de ce que sont la "bonne santé" et la médecine, sont des éléments intériorisés par l'individu et hérités socialement [11]. Ces éléments sont à prendre en compte pour pouvoir adapter les soins, mais ils sont souvent méconnus des soignants. Ils diffèrent selon les groupes sociaux et notamment selon les classes. Dans les classes populaires, le rapport au corps est défavorable en matière de santé ; il est moins conscient, moins médicalisé que dans les classes aisées [12]. La valorisation de la force physique, par exemple, pousse à nier les états de fatigue ou à répugner à se sentir malade. De même, la façon de verbaliser un symptôme, de localiser la souffrance, ou même de somatiser, est en partie déterminée par la socialisation ${ }^{1}$. À l'inverse, les classes aisées ont une perception et une verbalisation de ce qui a trait au corps, plus proches du monde médical.

Ces différences sociales sont à intégrer par les soignants. Un travail de traduction de la part de ceux-ci peut être nécessaire ; dès la demande de soins, alors que les populations les mieux dotées recourent aux institutions de soins dans un but précis, les plus démunies arrivent souvent à l'hôpital avec des demandes plus diffuses et des difficultés à exprimer leurs besoins. Ces obstacles sont encore plus importants chez les plus pauvres, pour qui la grande précarité s'accompagne d'un sentiment de honte générale, d'exclusion et de mésestime de soi, notamment chez les sans-abris [13,14].

I Au-delà des difficultés de communication avec le monde médical, le rapport au corps et à la santé engendre chez les plus démunis des renoncements aux soins et/ ou un recours tardif à ceux-ci. La précarité économique et la nécessité de faire face aux problèmes du quotidien entraînent des difficultés à se projeter dans l'avenir ; pourvoir aux besoins de base est souvent prioritaire par rapport à ceux liés à la santé. I Une attention diminuée est accordée au corps et au souci de soí, ce qui se manifeste concrètement par un recours aux soins souvent tardif dans un état avancé de la maladie et/ou une polypathologie, nécessitant alors le plateau technique de l'hôpital pour des soins lourds.

I Cet accès tardif aux soins s'explique également par un moindre recours à la prévention ou au dépistage [15], mais aussi par un suivi médical moins efficace en médecine de ville. En effet, les populations les plus pauvres vont moins souvent chez le médecin généraliste et

\section{Notes}

${ }^{1}$ La socialisation est le processus d'intériorisation tout au long de la vie des normes et des valeurs par un individu (notamment au travers de l'éducation). ${ }^{2}$ On notera que les bénéficiaires de la CMUc en principe ne sont pas concernés par le renoncement aux soins pour raison financière.

${ }^{3} \mathrm{La}$ CMUc ne rembourse pas les dépassements d'honoraires pratiqués par les médecins du secteur II.

${ }^{4}$ Pour aider à communiquer, entre autres avec les personnes non francophones, un kit de communication pour améliorer le dialogue entre les équipes soignantes et les patients est diffusé par l'Assistance publique des hôpitaux de Paris, aux éditions Elsevier Masson. 


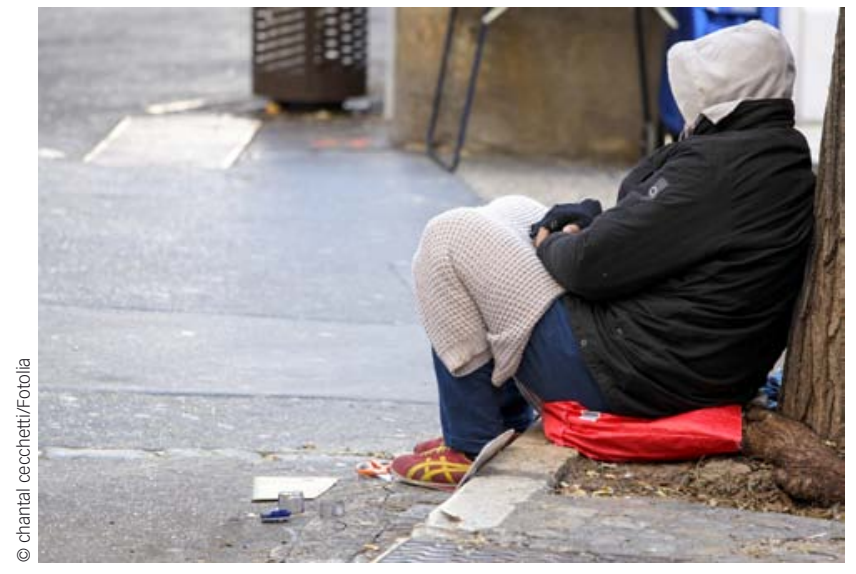

encore moins chez le spécialiste. Elles ont un moindre recours à la prévention (notamment aux tests de dépistage des cancers) et sont moins sensibles aux campagnes de prévention (alcool, tabac, alimentation, etc.) [16].

I Le renoncement aux soins, appelé le "renoncement barrière" [17], est souvent motivé par des raisons financières.

Il concerne les plus démunis qui disposent de faibles protections sociales (et a fortiori ceux qui n'en ont pas), comme par exemple les bénéficiaires de

\section{RÉFÉRENCES}

[1] Loi n ${ }^{\circ} 98-657$ du 29 juillet 1998 d'orientation relative à la lutte contre les exclusions, http://uww. legifrance.gouv.fr/affichTexte.do?ci dTexte=JORFTEXT000000206894\&c ategorieLien=id

[2] Rivollier E. Permanences d'accès aux soins de santé : une passerelle vers les personnes précarisées. La Santé de l'Homme (mars-avril) 2006;382:24-5.

[3] Geeraert J. Le socialà l'hôpital I'accès aux soins des populations en situation de grande précarité économique et sociale. Mémoire de recherche, Master 2 SPPS (Santé, Populations et Politiques Sociales), EHESS - Paris 13, sous la direction de Marie Jaisson. 2011, http://uww. appassra.org/theses-memoires/ [4] Organisation mondiale de la santé (OMS). Constitution de I'Organisation Mondiale de la Santé. 22 juillet 1946, http://uww.who.int/ governance/eb/who_constitution fr.pdf. cultés d'accès aux droits en matière de santé, soit liées à une incapacité à faire valoir des droits, soit à la complexité administrative des démarches. Les formulaires CMU, CMUc et AME compliqués à remplir ne peuvent souvent être réalisés qu'à l'aide d'une personne compétente pour décrypter le langage administratif. Ainsi sont confrontées aux démarches administratives les plus compliquées pour faire valoir des droits, les personnes les moins aptes à les surmonter. Ce problème est majeur lorsque la personne ne sait pas lire ou écrire [19]. D'autres préfèrent renoncer à une CMUc pour éviter le stigmate qu'elle représente, ou encore pour garder une liberté dans le choix de leur médecin traitant $^{3}$. Ainsi en 2006, 1,2 million de personnes n'ont pas fait valoir leur droit à la CMUc, soit un taux de non-recours de $20 \%$ de la population concernée par cette mutuelle [20].

I Par ailleurs, les délais d'attente pour l'obtention d'une assurance maladie peuvent être longs : trois à six mois pour une AME valable un an. Les étudiants connaissent aussi ce problème lorsqu'ils changent de caisse de Sécurité sociale. Fréquemment, des assurés bénéficiaires d'une CMU ou d'une AME se retrouvent sans couverture dans l'attente d'un renouvellement qui tarde du fait de la lourdeur et de la lenteur du travail administratif. Àces périodes de carence s'ajoutent des politiques, parfois discriminatoires, menées par les Caisses primaires d'assurance maladie (CPAM) concernant l'AME et la CMU, qui tendent à réduire ou à retarder l'accès aux droits [21, 22].

I En cas de problème de santé, la seule solution pour ces personnes, si elles sont infor- mées, consiste à se rendre dans une Pass ou dans des centres de soins gratuits du type de ceux de Médecins du monde. Beaucoup, désarmés face à cette complexité, ne sachant pas à qui s'adresser, essuyant plusieurs refus parce qu'ils n'étaient pas au bon endroit, ou exclus en raison de leur apparence, se découragent et arrivent à l'hôpital par la porte des urgences avec des pathologies aiguës et avancées [23]. Les bénéficiaires de la CMU et de l'AME connaissent également certains refus de soins de la part des médecins exerçant en secteur II, mais pas uniquement, d'où un frein supplémentaire à l'accès aux soins, surtout dans les grandes villes comme Paris.

\section{ÉCOUTER POUR REDONNER CONFIANCE}

Une demande de soins "vague" ou imprécise est très souvent une demande qui n'est pas formulée dans les termes médicaux attendus par les soignants. La première phase des soins consiste à prendre le temps de comprendre sa signification. Par ailleurs, chez les populations en situation de précarité, les demandes s'accompagnent souvent d'un besoin d'écoute et de contact social à ne pas négliger. Une prise en charge efficace sur le long terme requiert de l'empathie, de l'écoute et la création de lien social, surtout chez les personnes désocialisées ou en cours de désocialisation. Cette attitude soignante permet de leur redonner confiance en soi et de réduire leur méfiance concernant l'institution ou la médecine car ces patients se sentent rejetés par l'une ou par l'autre. La dévalorisation du care [24], le "prendre soin", est dommageable, surtout pour les 
personnes en grande précarité car c'est sur elles qu'il a le plus d'effets positifs en termes de santé.

I Le temps d'accueil, avant même le début des soins proprement dits, a une importance souvent méconnue par les services d'urgence. Une étude menée au Royaume-Uni [25] sur les soins de compassion auprès des sans-abris dans un service d'urgences a montré qu'un temps d'écoute autour d'un café, en plus des soins administrés habituellement, diminue le nombre moyen de passages aux urgences des patients concernés. Il permet notamment de diminuer leur méfiance vis-à-vis des institutions et de la médecine, crainte liée à de mauvaises expériences durant leur parcours biographique. Ainsi le carene se limite pas à l'acte technique ou médical, il intègre l'accueil, le dialogue, l'empathie et les soins sur le corps (pansements, nettoyage d'une plaie, etc.).

\section{Comprendre}

\section{POUR ADAPTER}

\section{LA DÉMARCHE SOIGNANTE}

【es situations précaires de ces personnes, où s'intriquent polypathologie, stade évolué de maladie, problèmes sociaux, souffrance psychosociale, ne sauraient se contenter des seuls soins techniques pratiqués sur les organes malades. Ces situations appellent une prise en compte des interdépendances qui existent entre maladie, mode de vie, rapport aux institutions, environnement social et culturel, rapport au corps et à la maladie.

Une prise en charge de qualité à I'hôpital nécessite de prendre le temps de connaître la situation du patient. Elle passe par la recherche de repères biographiques : son lieu de naissance, sa date d'installation en France, son travail, sa situation familiale, la nature de son logement (foyer, hébergement chez des amis ou la famille, chambre d'hôtel), son adresse postale ou l'adresse où la personne vit effectivement, sa situation administrative en matière de santé. Si la personne ne dispose pas de la Sécurité sociale, une orientation rapide vers un travailleur social $\mathrm{du}$ service ou vers la Pass de l'hôpital s'impose, car elle risque de ne pas avoir les moyens matériels et financiers de suivre le traitement prescrit. Ce temps, consacré au dialogue doit être compris comme faisant partie intégrante du soin. Loin d'être une perte de temps, il s'ouvre sur des décisions de soins davantage adaptées et réduit les errements de la prise en charge.

\section{COOPÉRER POUR INSCRIRE LES SOINS DANS LA DURÉE}

I Le mauvais état de santé général, l'intrication du social et du médical, la nécessité d'une prise en charge sur le Iong terme, sont des éléments imbriqués qui ne peuvent se résoudre par une seule prise en charge à l'hôpital. Une fois l'urgence médicale dédouanée ou traitée, les problèmes de santé non urgents, souvent aggravés par les conditions de vie, restent à considérer. Comme tous les maux ne peuvent être soignés à l'hôpital et les situations complexes être gérées par des soignants isolés, il est essentiel de travailler en collaboration avec les assistants sociaux qui pourront agir sur d'autres aspects de la vie du patient, notamment ceux non médicaux (logement, travail, nourriture, vêtements, etc.). En complément, le partenariat en réseau avec les associations et les services sociaux de secteur sont une aide à la prise en charge en amont et en aval des temps de soins hospitaliers [26]. Un décloisonnement entre travail social et travail de soins rend les actions des uns et des autres d'autant plus efficaces qu'elles se coordonnent et s'ajustent.

I Cependant, cette approche veille à ne pas décourager le patient. La prise en charge est réalisée par étapes, avec des objectifs peu nombreux sur le court ou le moyen terme, selon des priorités définies avec les personnes elles-mêmes qui éprouvent des difficultés à se projeter dans l'avenir. Des repères clairs sont posés dans le temps, l'espace, et face aux incertitudes de l'état de santé afin de guider et rassurer le patient.

I Pour une (ré)insertion dans le droit commun, en aval des soins hospitaliers, le travail est réalisé en partenariat avec un réseau de proximité : lien et/ou orientation vers des médecins libéraux, centres de santé, associations, centres médico-psychologiques de secteur, etc. Il est essentiel que chaque patient participe à ce cheminement ou, à défaut, donne son adhésion. Il s'agit tout d'abord de s'adosser aux points d'ancrage du patient, s'ils existent. Dispose-t-il d'un accompagnement social, éducatif ? Est-il déjà en lien avec des associations ? A-t-il un médecin "traitant" ? Désire-t-il conserver cet ancrage?

I La démarche, pour être fructueuse, s'appuie ainsi sur les ressources disponibles à l'intérieur comme à l'extérieur de I'hôpital, en sauvegardant les contacts et les informations utiles. Une prise en charge

\section{RÉFÉRENCES}

[5] Aïach P. Les inégalités sociales de santé. Écrits. Paris: Economica, Sociologiques; 2010. p. 43-77. [6] Paugam S. La disqualification sociale. Essai sur la nouvelle pauvreté. Paris: PUF; 1991. [7] Castel R. Les métamorphoses de la question sociale. Paris: Fayard; 1995.

[8] Paugam S. Le lien social. Paris: PUF, Que sais-je; 2009. [9] DubetF.Lagalère. Jeunes en survie. Paris: Fayard; 1987. [10] Fassin D, Morice A, Quiminal $C$ (dir.). Les lois de l'inhospitalité. Les politiques d'immigrationà l'épreuve des sans-papiers. Paris: La Découverte, Essais; 1997. [11] Mauss M. Les techniques du corps. Journal de Psychologie. 1935;32:271-93.

[12] Boltanski L. Les usages sociaux du corps. Les annales E.S.C. 1971;26:205-33.

[13] Ménoret-Calles B. L'accès aux soins des populations démunies. Situation et perspective en 1996. Paris: L'Harmattan, L'éthique en mouvement; 1997.

[14] De Gaulejac V. Les sources de la honte. Éditions Desclée de Brouwer; 2008 (1996).

[15] Dress. Le renoncement aux soins pour raisons financières. Analyse socio-anthropologique. Série Études et Recherche 2012;119.

[16] De Saint Pol T. La santé des plus pauvres. Insee Première (octobre); 2007. p. 1161.

[17] Questions d'économie de la santé (QES). Le renoncement aux soins : une approche socioanthropologique. 2011;169, http:// www.irdes.fr/Publications/2011/ Qes169.pdf

[18] Boisguerin B. Les allocataires des minima sociaux: CMU, état de santé et recours aux soins. Drees, Études et résultats (octobre) 2007;603.

[19] De Moerloose P.etal.

Éducation thérapeutique du patient et illettrisme en Suisse. Revue Médicale Suisse (6 février) 2008:323-324, www.revmed.ch [20] Dufour-Kippelen S, Legal A Wittwer J. Comprendre les causes de non-recours à la CMUc. Paris: Rapport de recherche LEGOS; 2006. [21] Gabarro C. Les demandeurs de l'aide médicale d'État pris entre productivisme et gestion spécifique. Revue Européenne des Migrations Internationales 2012;28/2:35-56. 


\section{RÉFÉRENCES}

[22] Martin P. Gestion de la file d'attente et invisibilisaton des précaires. Mensonge institutionnalisé dans une caisse d'assurance maladie. Actes de la recherche en sciences sociales 2011/4;189:34-41.

[23] De Goër B, Gales B, Agnoli A. Spécificités de la prise en charge d'un public précaire dans un service d'urgence. Société française de médecine d'urgence. Actes de congrès 2010, http://www. appassra.org/theses-memoires/ [24] Saillant F, Gagnon E. Présentation. Vers une anthropologie des soins?, Anthropologie et Sociétés 1999;23(2):5-14.

[25] Redelmeier DA. A randomised trial of compassionate care for the homeless in an emergency department. The Lancet 1995:345(8958):1131-4. [26] Rivollier E, Piegay E, ScanvionA. Exclusion, quelles prises en compte à l'hôpital ? Quelles liaisons avec le territoire? Revue hospitalière de France. 2013;550:41-6.

\section{Pour en SAVOIR PLUS}

- Conférence nationale de lutte contre la pauvreté et pour I'inclusion sociale. Travaux préparatoires. Santé et accès aux soins : pour un accès plus égal et facilité à la santé et aux soins. Novembre 2012, http://www. social-sante.gouv.fr/IMG/pdf/ sante_couv.pdf
Déclaration d'intérêts Les auteurs déclarent ne pas avoir de conflits d'intérêts en relation avec cet article. globale, la plus large possible, n'empêche pas de rester concentré sur le soin en cours : chacun reste dans son rôle, mais inscrit son action en appui et en relais d'autres professionnels.

\section{GARDER LES MÊMES RÈGLES PROFESSIONNELLES POUR TOUS}

Les soins des personnes en marge de notre système de santé, parce qu'elles sont désocialisées ou parce qu'elles appartiennent à des groupes étrangers à la culture des soignants hospitaliers, comportent des écueils. La crainte de heurter leur culture peut bloquer des soignants soucieux de la respecter. Une interaction réussie demande de la part du professionnel qu'il soit capable de mettre de côté ses propres représentations (préjugés et a priori) sur la culture de l'autre et qu'il sache rester dans son rôle en expliquant le pourquoi des soins proposés. Le patient garde son libre arbitre. Le soignant n'a pas à changer de culture, elle fait partie de son intégrité et d'ailleurs très souvent le patient ne la remet pas en cause ; la relation de soin est à établir dans la tolérance et le respect de la différence.

I Une personne en situation de précarité se présente souvent pour des soins en étant accompagnée d'un tiers qui la guide et la rassure ou qui aide à la traduction. Des biais dans la communication sont possibles, liés à la présence de ce tiers. Le soignant peut dégager un temps en tête-à-tête pendantl'examen clinique ou lors de gestes techniques. Dans tous les cas, il reste très prudent dans la relation avec le tiers, notamment dans la délivrance d'informations sur le diagnostic recherché ou établi. Il ne doit pas oublier que c'est le patient lui-même qui gère la révélation des informations soumises au secret médical. En revanche, un tiers peut être plus facilement associé aux démarches administratives et de soins, par exemple, pour s'orienter dans la ville ou l'hôpital, pour la tenue des rendezvous importants.

I'attitude des soignants, qui fait partie du langage des soins, est comprise par tous les patients quelle que soit leur provenance. Ainsi une attitude de démission aggrave l'angoisse d'un patient et renforce sa demande. Des attitudes respectueuses sont à maintenir même si le patient ne comprend pas notre langue ou semble exhiber sa déchéance.

I Le soignant doit garder à l'esprit que la précarité s'accompagne souvent d'une mauvaise connaissance du monde médical et que les personnes sont peu habituées aux médicaments et à l'observance d'un traitement. Dans la phase de décisions et de consignes, les explications données visent à être simples et logiques. Le soignant s'adapte au niveau de compréhension du patient et à ses conditions de vie. Il doit se centrer d'abord sur l'essentiel et sur les priorités, éviter les informations superflues et toute forme de sophistication. Il s'assure que le patient est bien d'accord pour la mise en œuvre des soins proposés, qu'il a bien compris les procédés de soins (préparation à tel examen complémentaire, ordonnance de médicaments, etc.). Il peut être aidé, par exemple à une bonne prise des médicaments, avec des signes portés sur l'ordonnance, des couleurs, ou encore le remplissage d'un pilulier'.

\section{ÉLARGIR LE CHAMP DES SOINS}

L'aide apportée au patient en situation de précarité à l'hôpital comprend une phase de protection de la personne pour éviter une complication d'une maladie, l'aider à obtenir une couverture sociale, à améliorer ses conditions de vie, à faire valoir ses droits, tout en prenant en compte d'éventuels troubles mentaux. Il s'agit d'arrêter le cercle vicieux des complications sociales et médicales. Cette aide vise une autonomie optimale de la personne en fonction de ses capacités. La façon d'accompagner ou de décliner l'aide doit toujours préserver la dignité de la personne.

La médecine est incapable de soigner seule des patients dont la situation sociale, environnementale ou psychologique a provoqué la pathologie, ou bien entravé le processus de recours aux soins. Les déterminants de la santé ne sont pas seulement physiques ou médicaux, mais également sociaux, psychologiques, culturels et environnementaux. Cela est d'autant plus vrai que les personnes se trouvent en bas de l'échelle sociale. Pour être efficace, leur prise en charge doit être globale et pluridisciplinaire et s'articuler avec le réseau extrahospitalier. Elle ne peut être réalisée sans prise en compte de leur souffrance psychosociale. Ainsi, un soignant amené à participer à la prise en charge d'une personne en situation de précarité, quel que soit le lieu d'exercice, élargira sa perception du soin et son savoir-faire pour mieux préserver la santé dans toutes ses composantes, notamment mentales et sociales. 